\title{
SUPERSETS FOR THE SPECTRUM OF ELEMENTS IN EXTENDED BANACH ALGEBRAS
}

\author{
MORTEZA SEDDIGHIN \\ Division of Natural Sciences \\ Lewis Clark College \\ Lewiston, ID 83501 \\ (Received June 1, 1987 and in revised form October 21, 1987)
}

ABSTRACT. If $\mathrm{A}$ is a Banach Algebra with or without an identity, $\mathrm{A}$ can be always extended to a Banach algebra $\bar{A}$ with identity, where $\bar{A}$ is simply the direct sum of $A$ and $\mathbb{C}$, the algebra of complex numbers. In this note we find supersets for the spectrum of elements of $\bar{A}$.

KEY WORDS AND PHRASES. Banach Algebra, spectrum of elements and quasi-singular elements.

1980 AMS SUBJECT CLASSIFICATION CODE. $46 \mathrm{H} 99$.

1. INTRODUCTION.

Let $A$ be a Banach algebra. Then we know that the set $\bar{A}=\{(x, \alpha): x \quad A, \alpha$ complex $\}$ together with the operations $(x, \alpha)+(y, \beta)=(x+y, \alpha+\beta)$ and $(x, \alpha)(y, \beta)=(x y+\beta x+\alpha y, \alpha \beta)$, and norm $\|(x, \alpha)\|=\|x\|+|\alpha|$ is a Banach algebra, whose identity element is $(0,1)$. Although this is usually done for algebras $A$ without identity, to extend them to algebras with identity; we can also start with a Banach algebra A with identity (In this case the identity of $A$ is no more an identity for $\bar{A})$.

2. MAIN RESULTS.

DEFINITION 2.1. An element $x$ in a Banach algebra $A$ is called quasi-regular if xoy $=$ yox $=0$ for some $y \in A$, where xoy $=x+y-x y$. xoy is called the circle operation. $x$ is called quasi-singular if it is not quasi-regular. For an element $x$ in $A$, the special radius of $x$ is defined by

$$
r(x)=\lim _{n \rightarrow \infty}\left\|x^{n}\right\|^{1 / n} .
$$

THEOREM 2,1. Let $\bar{A}$ be the extension of $A$, as above and let $\delta_{\bar{A}}((x, \alpha))$ denote the spectrum of $(x, \alpha)$ in $A$, then

$$
\delta_{\bar{A}}((x, \alpha)) \subseteq\{\alpha\} \cup \delta_{A}(x+\alpha),
$$

if $A$ already has an identity, and

$$
\delta_{\bar{A}}((x, \alpha)) \subseteq\{\lambda:|\lambda-\alpha| \leqq r(x)\}
$$


if $A$ does not have an identity.

PROOF. First suppose $A$ has an identity. Let $\lambda$ be a complex number, then $(x, \alpha)-(0, \lambda)=(x, \alpha-\lambda)$. If $\lambda \neq \alpha$, then

$$
(x, \alpha-\lambda)\left(y, \frac{1}{\alpha-\lambda}\right)=\left(x y+\frac{1}{\alpha-\lambda} x+(\alpha-\lambda) y, 1\right) .
$$

Now, if $\lambda \neq \alpha$ and $\lambda \notin \delta_{A}(x+\alpha)$, then the equation

$$
x y+\frac{1}{\alpha-\lambda} x+(\alpha-\lambda) y=0
$$

has a solution. To see this, write (2.1) as $(\alpha-\lambda) x y+x+(\alpha-\lambda)^{2} y=0$ or $(\alpha-\lambda)[x+\alpha-\lambda] y=-x$ or $y=\frac{1}{\alpha-\lambda}(x+\alpha-\lambda)^{-1}(-x) . \quad(x+\alpha-\lambda)^{-1}$ exists since $\lambda \notin \delta_{\mathrm{A}}(\mathrm{x}+\alpha)$.

This implies $\sim\{\alpha\} \cap \sim\left\{\delta_{A}(x)+\alpha\right\} \subseteq \sim \delta_{\bar{A}}((x, \alpha))$, and, therefore, we have:

$$
\delta_{\bar{A}}((x, \alpha)) \subseteq\{\alpha\} \cup \delta_{A}(x+\alpha)
$$

Now suppose $A$ does not have an identity and let $\lambda \neq \alpha$. If $\frac{1}{\alpha-\lambda} x$ is quasi-irregular, then there exists an element $z$ in $A$ such that:

$$
\frac{1}{\alpha-\lambda} x z+\frac{1}{\alpha-\lambda} x+z=0 \text {. }
$$

If we take $y=\frac{1}{\alpha-\lambda} z$, then we have:

$$
x y+\frac{1}{\alpha-\lambda} x+(\alpha-\lambda) y=0 \text {. }
$$

Hence, $\sim\{\alpha\} \cap \sim\left\{\lambda \mid \lambda \neq \alpha: \frac{1}{\alpha-\lambda} \quad x\right.$ is quasi-singular $\} \subseteq \sim \delta \frac{A}{A}((x, \alpha))$. For an element a in a Banach algebra, the inequality $r(a)<1$ implies a is a quasi-regular with quasiinverse $a=-\sum_{n=1}^{\infty} a^{n}$ (Rickart [1]). Hence, for an element $\frac{1}{\alpha-\lambda} \times$ to be quasisingular, it is necessary to have $r\left(\frac{1}{\alpha-\lambda} x\right) \geq 1$; that is $r(x) \geq|\lambda-\alpha|$

\section{REFERENCES}

1. RICKART, C.E. General Theory of Banach Algebras, D. Van Nostrand, Princeton, (1960). 


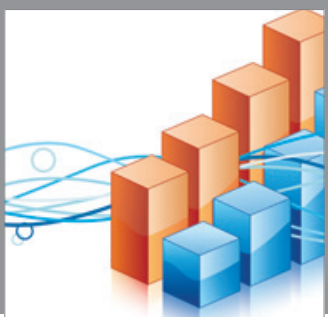

Advances in

Operations Research

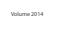

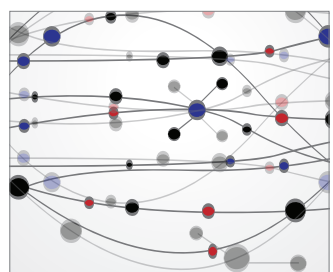

\section{The Scientific} World Journal
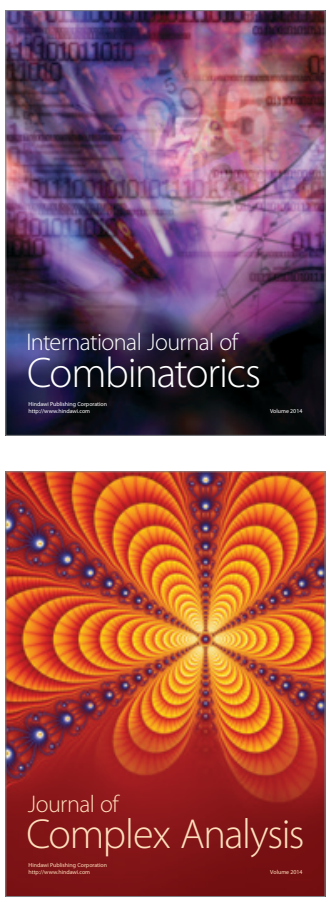

International Journal of

Mathematics and

Mathematical

Sciences
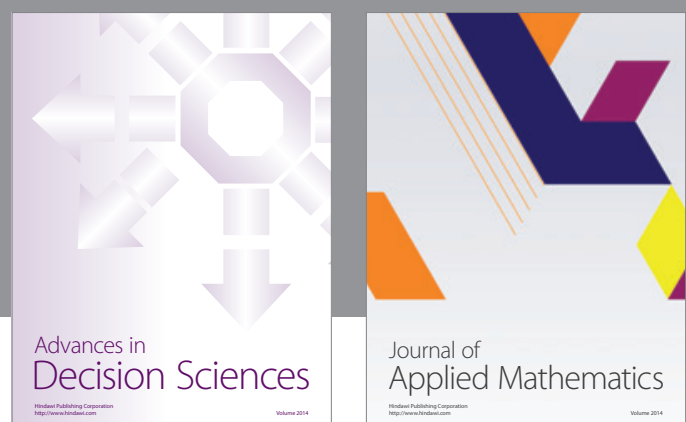

Journal of

Applied Mathematics
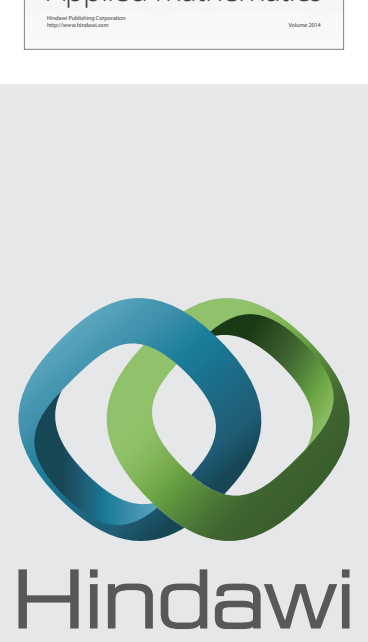

Submit your manuscripts at http://www.hindawi.com
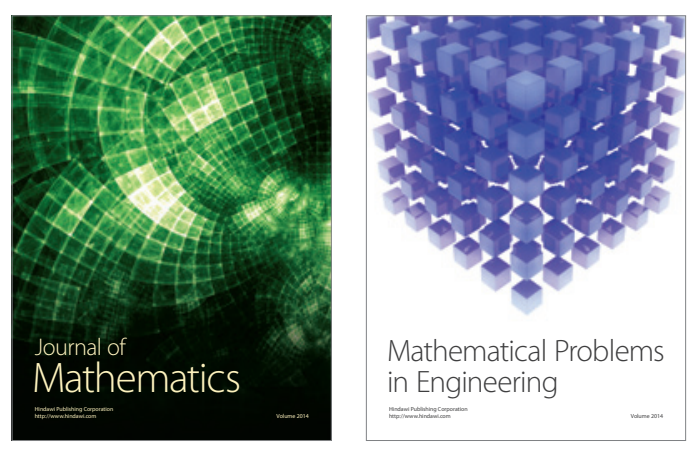

Mathematical Problems in Engineering
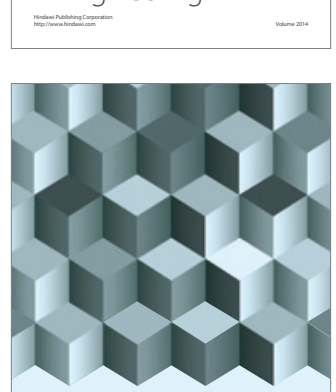

Journal of

Function Spaces
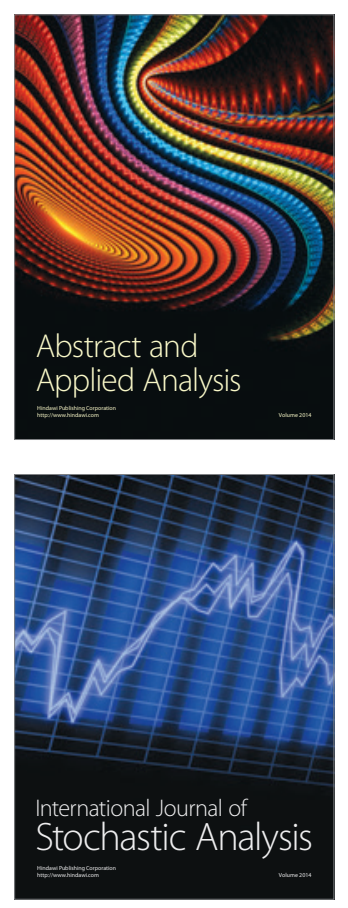

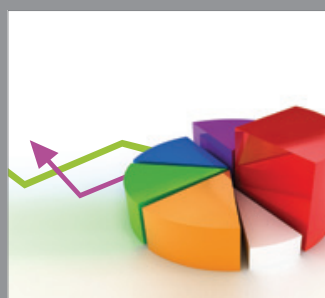

ournal of

Probability and Statistics

Promensencen
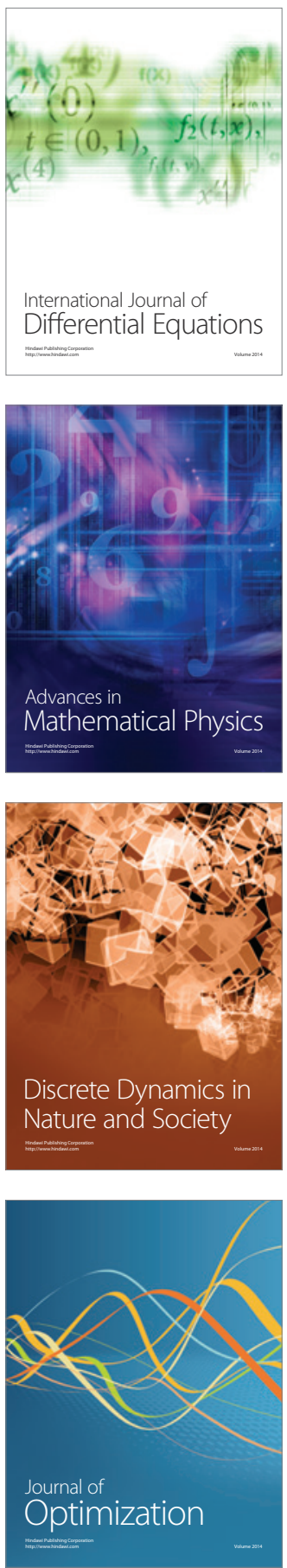